\title{
Predictive Motion Estimation for Reduced-Resolution Video from High-resolution Compressed Video
}

\author{
Justy W. C. Wong, Oscar C. Au, Peter H. W.Wong and A. Tourapis \\ Department of Electrical and Electronic Engineering, \\ The Hong Kong University of Science and Technology, \\ Clear Water Bay, Hong Kong, China \\ Email: eejusty@ee.ust.hk, eeau@ee.ust.hk, eepeter@ee.ust.hk, alexis@ee.ust.hk
}

\begin{abstract}
To convert a compressed video sequence to a lowerresolution compressed video, one typically needs to decompress the original sequence, down-sample each frame, and recompress it. It involves motion estimation in the reduced sequence which is computational intensive. In this paper, we propose a novel fast algorithm to predict the motion vector of the reduced video by using the original motion information in the compressed bitstream. We achieve a much higher quality than existing algorithms with low additional complexity.
\end{abstract}

\section{Introduction}

Nowadays, delivery of digital video through different kinds of network, such as internet, local area network, wireless network, cable TV network, is very common. Owing to the huge storage size of video, most stored video is only available in compressed format. For applications like previewing of a movie from Internet, transcoding to a lower bitrate in wireless network and down-conversion of HDTV video for low-resolution display, there is a need to reduce the resolution of the compressed video sequence which is stored in the video server and encode the reduced video into a compatible bitstream for the decoder.

In this paper, we are concerned of video compressed by ITU-T H.261, H.263, MPEG-1, MPEG-2. One thing common to these standards is that they employ motion estimation and compensation to reduce temporal redundancy between successive frames in a video sequence in order to achieve high compression efficiency.
A straight forward way to scale down the compressed video sequence is to decompress the whole sequence, apply anti-aliasing lowpass filtering, down-sample the video and then re-encode the reduced sequence by conventional video encoders which have to perform the computational intensive motion estimation again. If brute force motion estimation is used, more than $60 \%$ of the computational power of the encoder can be used by the motion estimation. Therefore, fast motion estimation is highly desirable.

In downscaling a video sequence from a frame size of $\mathrm{P} \times \mathrm{Q}$ to $\mathrm{P} / 2 \times \mathrm{Q} / 2$, a $16 \times 16$ macroblock in the reduced video corresponds to four $16 \times 16$ macroblocks in the original video. In Figure 1, it can
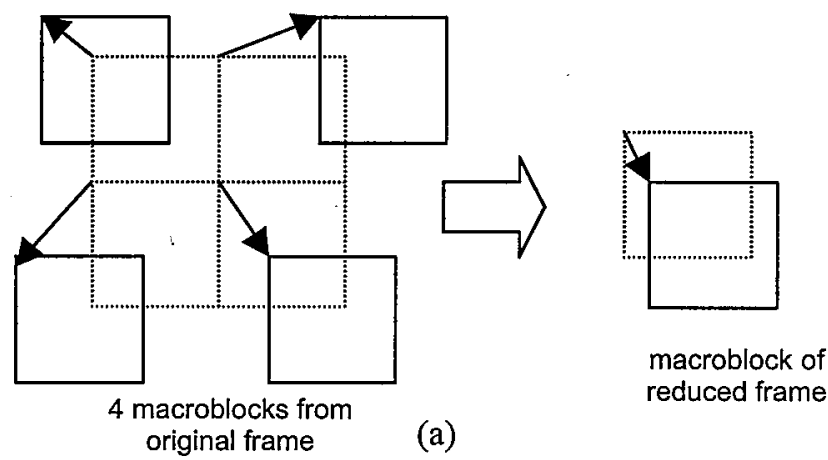

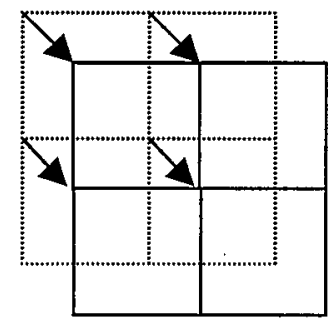

4 macroblocks from original frame
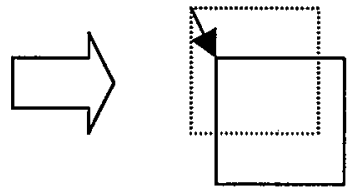

macroblock of reduced frame

(b)
Figure 1. Two possible relationships among motion vectors of the original frame and reduced frame. 
be seen that the motion vector of the macroblock in the reduced video is closely related to the motion vectors of the four macroblocks in the original video. As the original motion information is available in the compressed bitstream and should be highly correlated to the motion of the reduced video, it is possible to estimate the new motion vectors from the original motion vectors in order to save much of the computational cost. There are some existing algorithms for this propose but they do not perform very well.

In this paper, we propose a new motion estimation algorithm called predictive motion estimation (PME) to approximate the optimal motion vector for reduced video sequence using the motion information of the original compressed video sequence. We will show that PME is much better than the existing algorithms.

\section{Existing Algorithms}

Let $\left\{\mathrm{MB}_{o, i}\right\}_{i=1}^{4}$ be four macroblocks of the original video with corresponding motion vectors $\left\{V_{o, i}\right\}_{i=1}^{4}$. The four macroblocks are reduced to form one macroblock $\mathrm{MB}_{r}$ of the reduced video with corresponding motion vector $V_{r}$.

The simplest algorithm of getting the new motion vectors is to take the mean of the motion vectors of the four corresponding macroblocks in the original video and reduce it by one half and we call this MEAN which can be represented by the following equation:

$$
V_{r}=\frac{1}{2} \text { truncate }\left(\frac{\sum_{i=1}^{4} V_{o, i}}{4}\right)
$$

The MEAN is a good estimate if the four macroblocks have motion vectors pointing to similar directions with similar magnitude, as in the case of Fig. 1b. However, if the four motion vectors have different directions and/or magnitude, the resulting MEAN can be poor and meaningless as in the case of Fig. 1a. The simple arithmetic mean of the four different motion vectors is inadequate to describe all situations. In [2], an algorithm called adaptive motion vector resampling (AMVR) was proposed to estimate the motion vector of the reduced video using a weighted mean of the original motion vectors.

In the compressed video bitstream of MPEG-1, MPEG-2, H.261 and H.263, only the inter-coded macroblocks in the interframes ( $\mathrm{P}$ or $\mathrm{B}$ frames) contain motion vector information. AMVR applies only to these inter-coded blocks. AMVR uses a weighted mean of the four $V_{o}$ to estimate $V_{r}$.

$$
V_{r}=\frac{1}{2} \frac{\sum_{i=1}^{4} V_{o, i} \cdot A_{i}}{\sum_{i=1}^{4} A_{i}}
$$

where $A_{i}$ denotes the activity measurement of residual macroblock $i$ (in the original video). In [2], the number of non-zero AC coefficients was used as $A_{i}$. The complexity of AMVR is also very low but it is not necessarily good. Actually, it gives a rather low peak signal-to-noise ratio (PSNR) when compared with full search in the reduced video as we will show in section 4 .

\section{Predictive Motion Estimation (PME)}

Here we propose a new algorithm to predict the motion vector for the reduced video with significantly higher accuracy than AMVR. We will construct a weighted motion vector different from AMVR as a candidate. We will also consider the four original motion vector as four other candidates. All five candidates will be tested in search of the best motion vector.

We observe that in many cases the motion vector of a $\mathrm{MB}_{r}$ in the reduced video found by full search is similar to one or more of the four original motion vectors reduced by half. In these cases, taking the mean or weighted mean is usually inappropriate. In addition, all four original motion vectors appear to be equally likely. They should all be considered possible candidates.

For each reduced macroblock, if the 4 reference $V_{o}$ $\left(V_{o, 1}, V_{o, 2}, V_{o, 3}, V_{o, 4}\right)$ are the same, we will just take the common value and reduce it by half as the $V_{r}$. Otherwise, we will go through the following steps to get the $V_{r}$. 
Step 1: Compute 4 candidate vectors $\left(V_{r, i}\right)$ as

$$
V_{r, i}=\operatorname{truncate}\left(V_{o, i}\right) / 2
$$

The $V_{r, i}$ will be in half pixel precision.

$$
\text { for } i=1,2, \ldots 4
$$

Step 2: Compute the mean absolute difference $\left(M A D_{i}\right)$ in the reduced video for each of the four candidates $\left\{V_{r, i}, i=1,2, \ldots 4\right\}$. If one of the $M A D_{i}$ value is equal to zero, assign the corresponding $V_{r, i}$ as the new $V_{r}$ and stop searching. Otherwise, go to step 3.

Step 3: $\quad$ Compute a new candidate vector $V_{r, 5}$ by

$$
V_{r, 5}=\frac{\sum_{i=1}^{4}\left[V_{r, i} \cdot\left(\frac{1}{M A D_{i}}\right)\right]}{\sum_{i=1}^{4}\left(\frac{1}{M A D_{i}}\right)}
$$

Step 4: If the $V_{r, 5}$ is not equal to one of the first 4 candidates, compute $M A D_{5}$ using $V_{r, 5}$ as the motion vector.

Step 5: The $V_{r, i}$ with the minimum MAD value is chosen as the final $V_{r}$.

We call this algorithm predictive motion estimation (PME). For the $\mathrm{MB}_{o}$ that are skipped blocks or intra blocks which do not provide any motion information, we set the $V_{o}$ of these blocks to be zero.

For better performance, we can perform an optional additional step. We can search the 8 surrounding full or half pixel of the optimal $V_{r}$ found by PME.

The PME with a local full pixel search will be PMELS1. The PME with a local half pixel search will be called PME-LS2. Both algorithms have the following addition step.

Step 6: Compute the MAD for the surrounding 8 full pixels or half pixels. The one with the minimum $M A D$ will be the new motion vector $V_{r}$.

Note that step 6 is needed anyway if half pixel accuracy is needed in the full search.

\section{Results}

We tested our algorithms on several MPEG I video sequence with CIF resolution $(352 \times 288)$ and a GOP of 15 frames with IPPP frame structure in each GOP. The reduced-resolution sequence is in QCIF resolution $(176 \times 144)$. In order to compare the performance of different algorithms, we calculate the PSNR between the predicted frames of the reduced video using different algorithms and the original reduced frames.

Figure 2 shows the PSNR difference from full search of AMVR, MEAN and PME-LS1 for the "football" sequence. The search area for full search is \pm 7 .

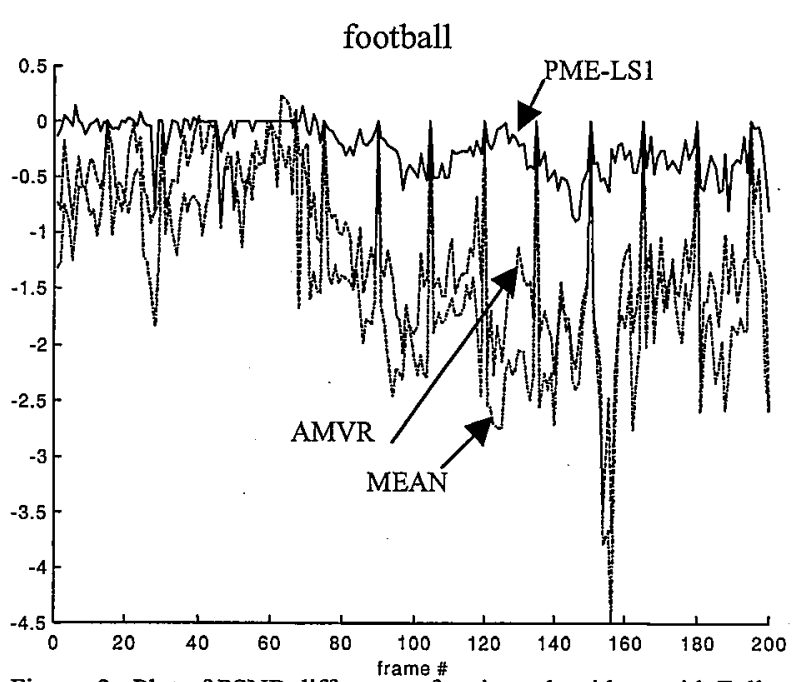

Figure. 2. Plot of PSNR difference of various algorithms with Full search

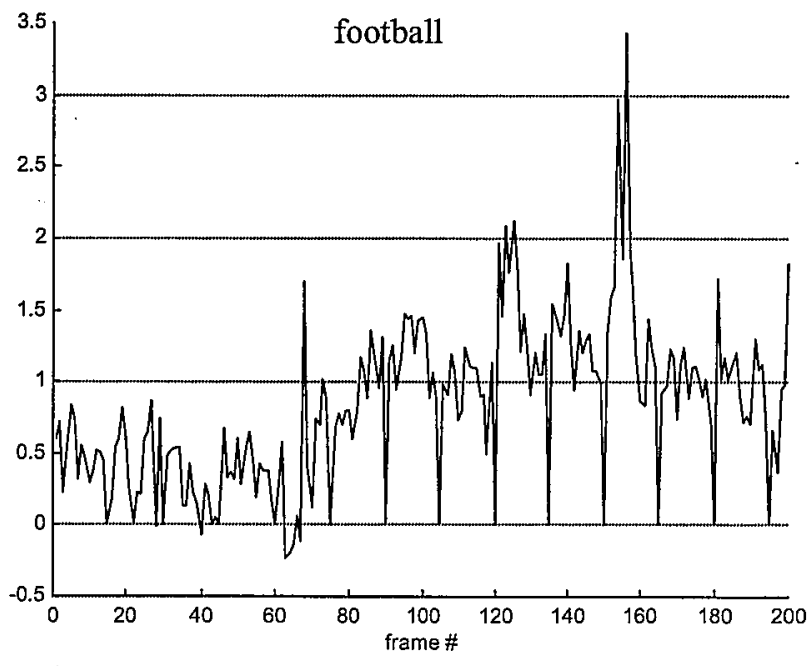

Figure 3. Plot of PSNR difference between PME-LS1 and AMVR 
Figure 3 shows the PSNR difference between PMELS1 and AMVR in football sequence. It shows a maximum of about $3.5 \mathrm{~dB}$ improvement over AMVR and an average of $0.8388 \mathrm{~dB}$.

Table 1 shows the mean PSNR values of different algorithms, the difference in mean PSNR values between full search and different algorithms and the average search point for each macroblock for the "football" and "salesman" test sequences. For the fast moving scene, it seems that PME-LS1 has better performance than PME-LS2. However, for the headand-shoulder scenes, PME-LS2 is better.

If we use the PME without step 3 and step 4, i.e. without the $V_{r, 5}$ (we call this PV4 in Table 1), the PSNR will be much lower than PME. This shows that the fifth candidate $V_{r, s}$ is very important.

In PME, we calculate the MAD for at most 5 points for each macroblock and do not need to calculate for those blocks with four identical motion vectors. In the case of the fast moving scenes in football sequence, the average search points for each motion vector is about 0.6 which is much less than full search of 225 search points. In the case of the headand-shoulder scene like salesman, the average search point is about 0.016 . We can achieve a even better quality with the additional local search in PME-LS1 or -LS2 at the price of more search points.

\section{Conclusions}

In this paper, we propose a fast algorithm to estimate the best motion vector for reduced resolution video sequences using the motion information of the original compressed video sequence. The computational need of the proposed algorithm is minimal and the prediction is much better than AMVR in terms of PSNR.

\section{Acknowledgement}

This work was funded by RGC grant HKUST6009/97E.

\section{References}

[1] B. Natarajan and V. Bhaskaran, "A fast approximate algorithm for scaling down digital images in the DCT domain," Proc. Of IEEE Int. Conf. On Image Processing, Oct. 1995.

[2] B. Shen, I. K. Sethi and V. Bhaskaran, "Adaptive motion vector resampling for compressed video down-scaling," Proc. Of IEEE Int. Conf. On Image Processing, Oct. 1997.

\begin{tabular}{|c|c|c|c|c|c|c|}
\hline \multirow[t]{2}{*}{ Sequence } & \multicolumn{3}{|c|}{ Football } & \multicolumn{3}{|c|}{ Salesman } \\
\hline & $\begin{array}{l}\text { Mean } \\
\text { PSNR }\end{array}$ & $\begin{array}{c}\text { PSNR } \\
\text { difference }\end{array}$ & $\begin{array}{c}\text { Average Search } \\
\text { points }\end{array}$ & $\begin{array}{l}\text { Mean } \\
\text { PSNR }\end{array}$ & $\begin{array}{c}\text { PSNR } \\
\text { difference }\end{array}$ & $\begin{array}{c}\text { Average Search } \\
\text { points }\end{array}$ \\
\hline Mean only & 22.2758 & -1.8317 & * & 35.8721 & -1.1519 & * \\
\hline AMVR & 22.6499 & -1.4576 & $*$ & 35.9382 & -1.0857 & * \\
\hline PV4 & 22.8599 & -1.2476 & 0.6551 & 36.2721 & -0.7521 & 0.01643 \\
\hline PME & 23.3042 & -0.8033 & 0.6597 & 36.9447 & -0.0792 & 0.0169 \\
\hline PME-LS1 & 23.5418 & -0.5657 & 5.937 & 36.9519 & -0.0721 & 0.1521 \\
\hline PME-LS2 & 23.4887 & -0.6188 & 5.937 & 36.996 & -0.028 & 0.1521 \\
\hline Full search & 24.1075 & 0 & 225 & 37.0242 & 0 & 225 \\
\hline
\end{tabular}

Table 1. mean PSNR and mean PSNR difference from full search for various algorithms and various test sequences.

* These two algorithms have negligible computation compared with full search. 